\title{
Neutron resonance transmission analysis of cylindrical samples used for re- activity worth measurements
}

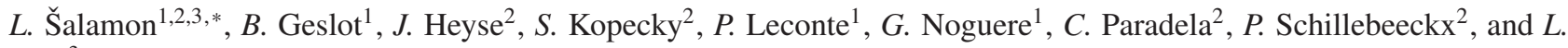 \\ $\mathrm{Snoj}^{3}$ \\ ${ }^{1}$ DER, DEN, CEA, Cadarache, F-13108 Saint-Paul-lez-Durance, France \\ ${ }^{2}$ EC-JRC-Geel, B-2440 Geel, Belgium \\ ${ }^{3}$ Reactor Physics Division, Jozef Stefan Institute, SI-1000 Ljubljana, Slovenia
}

\begin{abstract}
A characterisation of cylindrical samples by Neutron Resonance Transmission Analysis (NRTA) at the GELINA facility of JRC Geel (Belgium) is presented. The samples were designed and produced for reactivity worth measurements in the MINERVE reactor of CEA Cadarache (France). NRTA was applied to determine the nuclide composition of $\mathrm{UO}_{2}, \mathrm{Al}_{2} \mathrm{O}_{3}$ and liquid samples that were doped with silver. The volume number densities of ${ }^{238} \mathrm{U},{ }^{107} \mathrm{Ag}$ and ${ }^{109} \mathrm{Ag}$ obtained by NRTA are within $2 \%$ fully consistent with the values that are quoted by the manufacturer. In addition, the NRTA data reveal a tungsten contamination which is not reported by the provider. It is shown that such a contamination contributes by up to $5.7 \%$ to the reactivity worth.
\end{abstract}

\section{Introduction}

The MINERVE facility, a zero-power reactor located at the CEA Cadarache (France), operated from 1959 to 2017. It was designed for reactivity worth measurements by the pile-oscillation technique, that is, by oscillating small samples in the centre of the reactor core. The experimental programs carried out in MINERVE were dedicated to measure neutron absorption cross section of specific elements in a well-characterised neutron field. The samples were cylinders with a length of $10 \mathrm{~cm}$ and an outer diameter of about $1 \mathrm{~cm}$, as shown in Fig. 1. Hundreds of samples containing small amounts of actinides, fission products, structural materials or neutron absorbers, were produced. Final results given were the ratios between the calculated reactivity worth value $\mathrm{C}$ and experimental reactivity worth value $\mathrm{E}$ (i.e. $\mathrm{C} / \mathrm{E}$ ratios). An analysis of the experiments at MINERVE reveals inconsistencies of a few percent between the calculated and experimentally determined reactivity worth. The observed differences cannot be explained by erroneous neutron cross sections used in the calculations [? ]. Although the sample composition provided by the manufacturer was determined by radiochemical analysis, bias effects due to the sample composition are not excluded. In this work the sample composition, in particular the presence of neutron absorbing elements, is verified by Neutron Resonance Transmission Analysis (NRTA).

\section{MINERVE sample characteristics}

Five MINERVE samples, referred to as AG9C1, AG9C2, UNC, AAG and MAG, were studied. They were used in the framework of the Burn-Up Credit (BUC) and MAESTRO programmes at the MINERVE facility [0], [2]. The AG9C1 and AG9C2 samples consist of a stack of ten pellets enclosed in two Zircaloy-4 (Zy-4) containers. The pellets were manufactured by mixing depleted UO2 powder with silver with different enrichments in ${ }^{109} \mathrm{Ag}$. The performance of NRTA to determine the amount of uranium was assessed by measurements of a reference sample containing only $\mathrm{UO}_{2}$. This sample is referred to as UNC. In addition, a sample consisting of an $\mathrm{Al}_{2} \mathrm{O}_{3}$ matrix doped with natural silver was measured (AAG). This sample has the same design and manufacturing characteristics as the AG9C1 and AG9C2 samples. Finally a liquid sample, referred to as MAG, which was measured in the MAESTRO programme, was characterised by NRTA. This sample consists of natural silver dissolved in a wellcharacterised $4 \% \mathrm{HNO}_{3}$ solution. The solution was enclosed in a double watertight cladding: one made of a welded Zy-4 alloy and one made of an Al-5754 alloy.

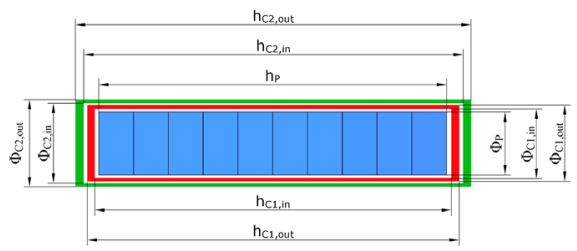

Figure 1. Technical drawing of the MINERVE samples, consisting of a ten-pellet stack (in blue) contained in two Zircaloy-4 containers (in red and green).

*e-mail: linosalamon@hotmail.com 


\section{Neutron transmission measurements}

The NRTA experiments were performed at the time-offlight (TOF) facility GELINA located at the JRC Geel [3]. Measurements were carried out at flight path 13, at a distance of $10.9 \mathrm{~m}$ from the neutron production target. This flight path forms an angle of $18^{\circ}$ with respect to the direction normal to the exit face of the moderator. The MINERVE samples were mounted in an automatically controlled sample changer. The samples were positioned with the cylindrical axis of the sample oriented vertically with respect to the direction of the neutron beam. The neutron beam was collimated to a diameter of about $15 \mathrm{~mm}$ at the sample position. Consequently, for the MINERVE samples the neutron beam diameter is larger than the sample diameter, which is about $10 \mathrm{~mm}$. Two types of antioverlap filters, i.e. $\mathrm{a}^{10} \mathrm{~B}$ and $\mathrm{Cd}$ filter, were used to reduce the contribution of slow neutrons coming from previous accelerator pulses. A fixed cobalt and sodium black resonance filter were placed in the beam to continuously control the background level at $132 \mathrm{eV}$ and $2850 \mathrm{eV}$, respectively. Neutrons were detected with a $6.4 \mathrm{~mm}$ thick Liglass scintillation detector, enriched to $95 \%$ in ${ }^{6} \mathrm{Li}$. The scintillator was coupled to an EMI9823-QKB photomultiplier (PMT) using a quartz entrance window. The TOF of a registered neutron $t$ is derived from the difference between a stop signal $\mathrm{T}_{s}$, obtained from the anode signal of the PMT, and a start signal $\mathrm{T}_{0}$ that is produced by each electron burst. This time difference needs to be corrected for a time-offset, which is mainly due to a difference in cable lengths used for the recording of $\mathrm{T}_{0}$ and $\mathrm{T}_{s}$. The time difference $\mathrm{T}_{S}-\mathrm{T}_{0}$ is measured with a multi-hit fast time to digital converter (TDC). The data reduction process was performed with the AGS code [4], developed at the JRC Geel. The experimental transmission is mostly derived from the ratio of the TOF spectra taken with and without a sample, both corrected for dead time and background. For measurement in a good transmission geometry, i.e. a parallel incident neutron beam, a homogeneous sample and a fixed geometrical neutron path length through the sample, this experimental ratio can be directly related to the areal density and total cross section through the Lambert-Beer law. The geometry for the measurements of the cylindrical MINERVE samples does not fulfil the good transmission geometry conditions. Part of the neutron beam does not interact with the sample and the geometrical path length of the neutrons in the sample varies (Fig. 2). Ideally these effects are included in the calculation of the theoretical transmission when performing a resonance shape analysis (see e.g. Ref. [5]). In this work an experimental transmission $T_{\text {exp }}^{\prime}(t)$ is derived which is corrected for the fraction of the neutron beam that is not covered by the sample:

$$
T_{\text {exp }}^{\prime}(t)=N_{T} \frac{\left[C_{\text {in }}(t)-B_{\text {in }}(t)\right]-\alpha\left[C_{\text {out }}(t)-B_{\text {out }}(t)\right]}{(1-\alpha)\left[C_{\text {out }}(t)-B_{\text {out }}(t)\right]},
$$

where $\alpha$ is the void fraction. The sample-in and sampleout TOF spectra are denoted by $C_{\text {in }}$ and $C_{\text {out }}$, respectively, and their corresponding background contributions by $B_{\text {in }}$ and $B_{\text {out }}$. For samples covering the full area of the neutron beam such that $\alpha=0$, the expression in Eq. 1 reduces to the one used for data taken in good transmission geometry. The spectra $\left(C_{\text {in }}, C_{\text {out }}, B_{\text {in }}, B_{\text {out }}\right)$ are corrected for losses due to the dead time in the detection system, normalised to the TOF bin width and to the integrated count rate of the $\mathrm{BF}_{3}$ counters. The normalisation factor $\mathrm{N}=1.0000$ (25) accounts for the uncertainty from the variations in the beam intensity and electronics. The uncertainty due to such variations is reduced to less than $0.25 \%$ by alternating sequences of sample-in and sample-out measurements. The background as a function of TOF is approximated by an analytical function [6] with the parameters adjusted to the black resonance dips due to the presence of the fixed $\mathrm{Co}$ and $\mathrm{Na}$ filters. A sample-in TOF spec-

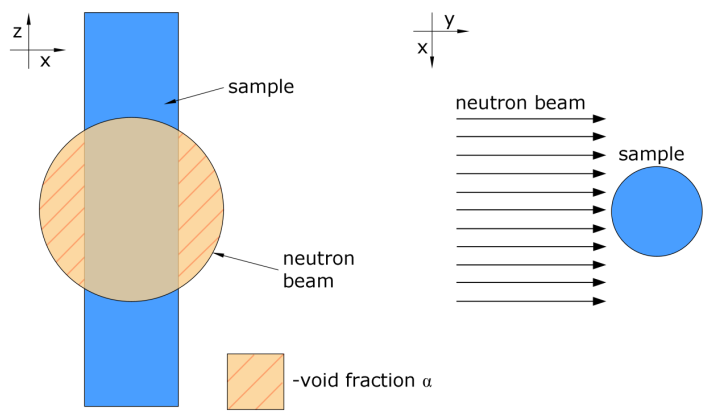

Figure 2. Schematic presentation of the MINERVE sample in the neutron beam.

trum resulting from the transmission measurements with the AG9C1 sample is shown in Fig. 3. This figure reveals that the amplitudes of the dips for the black resonances due to nuclides present in the sample, e.g. the dips at the 5.2 $\mathrm{eV}$ resonance of ${ }^{109} \mathrm{Ag}$ and the $6.7 \mathrm{eV}, 20.9 \mathrm{eV}$ and 36.7 $\mathrm{eV}$ resonances of ${ }^{238} \mathrm{U}$ resonances, are less compared to those due to the presence of the fixed black resonance filters, which fully cover the beam. This difference is due to the void fraction, which can be estimated by comparing the transmission dips of the ${ }^{109} \mathrm{Ag}$ and ${ }^{238} \mathrm{U}$ black resonances with the value expected from the background derived from measurements with filters fully covering the beam.

\section{Neutron Resonance Transmission Analysis with the REFIT code}

The composition of the sample was derived by a least squares fit to the data using the resonance shape analysis code REFIT [7]. This code is optimised to determine resonance parameters by comparing the experimental transmission with the theoretical one. It accounts for various experimental effects such as Doppler broadening and response function of TOF spectrometer. The theoretical transmission as a function of the incident neutron energy in case of a homogeneous sample and a constant neutron path length is given by the Lambert-Beer law, which is not valid for the MINERVE samples with cylindrical geometry. Therefore, a new analytical expression was implemented in the REFIT. The transmission through a cylindrical sample starting from an incident parallel, uniform 


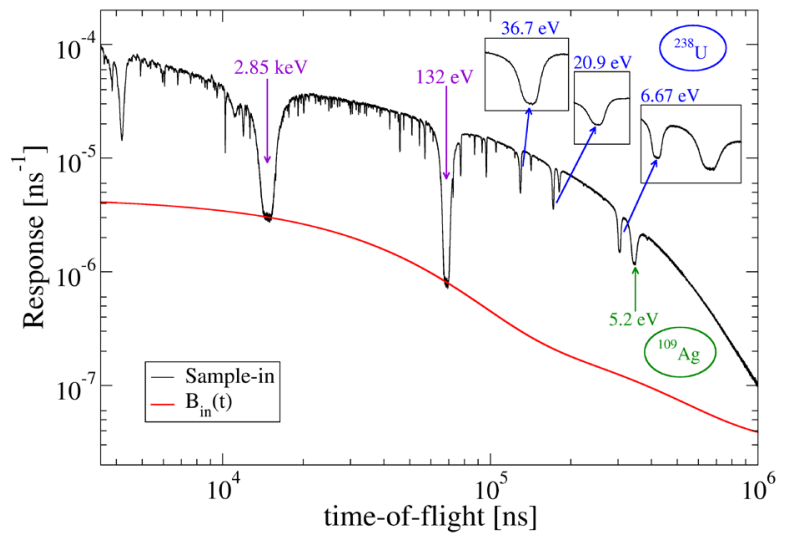

Figure 3. Sample-in spectra from the transmission measurement with the AG9C1 sample. Data are compared with the total background (red line). Black resonances due to the presence of the fixed black resonance filters and black resonances due to nuclides present in the sample are indicated.

neutron beam is expressed as:

$T^{\prime}(E)=\frac{\int_{-r}^{r} \sqrt{1-(x / R)^{2}} \exp \left[-2 r \sqrt{1-(x / r)^{2}}\left(\sum_{k} \rho_{k} \bar{\sigma}_{t o t, k}(E)\right)\right] d x}{\int_{-r}^{r} \sqrt{1-(x / R)^{2}}}$

where $\rho_{k}$ is the volume number density of nuclide $k, \bar{\sigma}_{t o t, k}$ its Doppler broadened total cross section and $r$ and $R$ are the sample and beam radius, respectively. The analytical model in Eq. 2 neglects the attenuation in the cladding material. This contribution is in first approximation energy independent and is lumped in an adjustable normalization factor. The analytical expression of Eq. 2 was validated by Monte-Carlo simulations using the MCNP 5 code [8]. A simplified AG9C1 sample made of ${ }^{109} \mathrm{Ag}$ and ${ }^{238} \mathrm{U}$ was considered. The transmission through the sample was calculated for a parallel uniform neutron beam without any void fraction. The flat residual in Fig. 4 shows a good agreement between the MCNP simulation and the REFIT calculation. In ideal conditions, that is, for a uniform parallel neutron beam that is perpendicular to the sample, the sample radius $\mathrm{r}$, the beam radius $R$ and void fraction $\alpha$ are linked by the expression:

$$
\alpha=1-\frac{2 R^{2} \arcsin (r / R)+2 r \sqrt{R^{2}-r^{2}}}{\pi R^{2}} .
$$

In practice, the neutron beam intensity is not uniformly distributed in both the radial and vertical direction. The distribution in vertical direction is even asymmetric. To account for the non-uniformity of the neutron beam the sample and beam radius are replaced by effective radii in the analysis. An effective sample radius $r_{e f f}$ is adjusted to the resonance dips in the experimental transmission in the region of ${ }^{238} \mathrm{U}$ resonance at $80.7 \mathrm{eV}$. A corresponding effective beam radius $\mathrm{R}_{\text {eff }}$ is derived from Eq. 3 using the experimentally defined void fraction. The MAG liquid sample has a larger radius and does not contain ${ }^{238} \mathrm{U}$. Therefore, the effective sample radius was derived from a fit to the dip due to the $55.8 \mathrm{eV}$ resonance of ${ }^{107} \mathrm{Ag}$ using the parameters reported in Ref. [9].

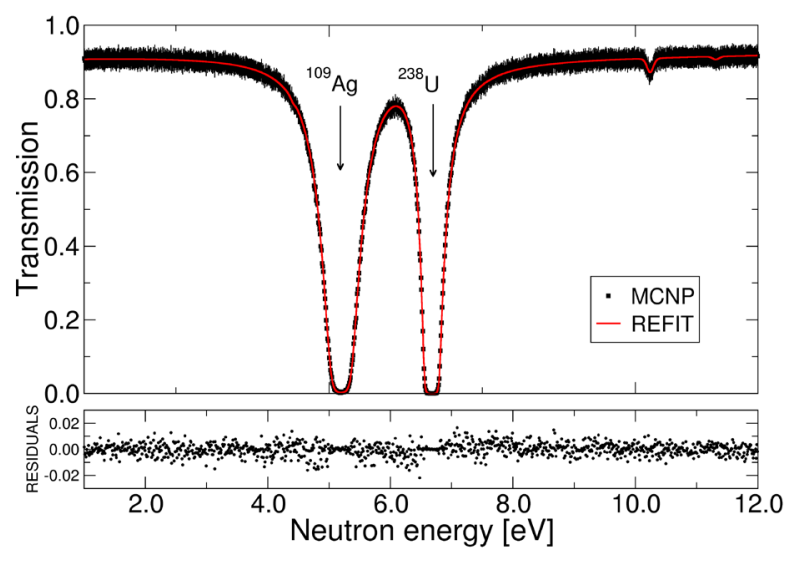

Figure 4. Comparison between the MCNP simulation and the REFIT calculation. Residuals given below each plot are calculated as the difference between the data coming from the MCNP simulation and the REFIT calculation.

\section{Nuclide composition of the MINERVE samples}

NRTA was applied to determine the composition of the samples and to compare with the one provided by the manufacturer based on ICPMS. The volume number density of nuclides showing resonance dips were derived from a least squares adjustment to the experimental transmission using the model described by Eq. 2. For the analysis the ${ }^{238} \mathrm{U}$ resonance parameters based on the work of Derrien et al. [10] and the ${ }^{107,109} \mathrm{Ag}$ parameters reported in Ref. [9] were taken. Most of the resonance energies were also adjusted to improve the results of the fit. Fig. 5 shows the experimental and calculated transmission for incident neutron energies smaller than $100 \mathrm{eV}$. For relatively weak resonances, a good agreement between the experimental and calculated transmission is obtained. In the vicinity of the black resonances, structures are observed in the residuals indicating that the model does not fully describe the data. This could be related to the assumption of a parallel and uniform neutron beam. The differences between the NRTA results and values declared by the provider reported in Table 1 are within $2 \%$. These results confirm that NRTA can be used to determine the composition of cylindrical samples.
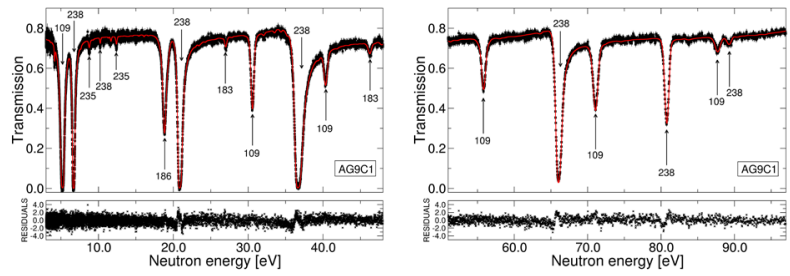

Figure 5. Transmission spectra through the AG9C1 are compared with the theoretical REFIT calculation using Eq. 2.

Some of the resonance dips in the transmission resulting from the measurements with the AG9C1, AG9C2, 
Table 1. The ratios of $\left({ }^{107,109} \mathrm{Ag}\right.$ and $\left.{ }^{238} \mathrm{U}\right)$ volume number densities derived from the NRTA to the volume number densities provided by the manufacturer.

\begin{tabular}{llll}
\hline Sample & ${ }^{107} \mathrm{Ag}$ & ${ }^{109} \mathrm{Ag}$ & ${ }^{238} \mathrm{U}$ \\
\hline AG9C1 & - & $1.019(33)$ & $1.022(38)$ \\
AG9C2 & - & $0.991(16)$ & $1.010(42)$ \\
AAG & $0.990(12)$ & $1.002(28)$ & - \\
MAG & $1.009(34)$ & $1.008(26)$ & - \\
\hline
\end{tabular}

AAG and UNC samples cannot be related to resonances of the nuclides listed in the sample composition provided by the manufacturer. Most of these dips can be linked to resonances of natural tungsten. A common point of the samples is the manufacturing process of the pellets by powder compacting technique. For the grinding step, tungsten carbide balls were used as a grinding material. The NRTA results suggest that tungsten observed in the pellets comes from a malfunction of the grinder. If the contamination of the MINERVE samples is due to the erosion of grinding material, it can be assumed that tungsten is homogeneously distributed in the sample. The transmission dip due to the $18.8 \mathrm{eV}$ resonance was used to fit the amount of ${ }^{186} \mathrm{~W}$ (see Fig. 6). The amount of the other isotopes was obtained from the natural abundance of tungsten. The volume number densities for the different samples indicate that the tungsten contamination strongly varies from sample to sample.

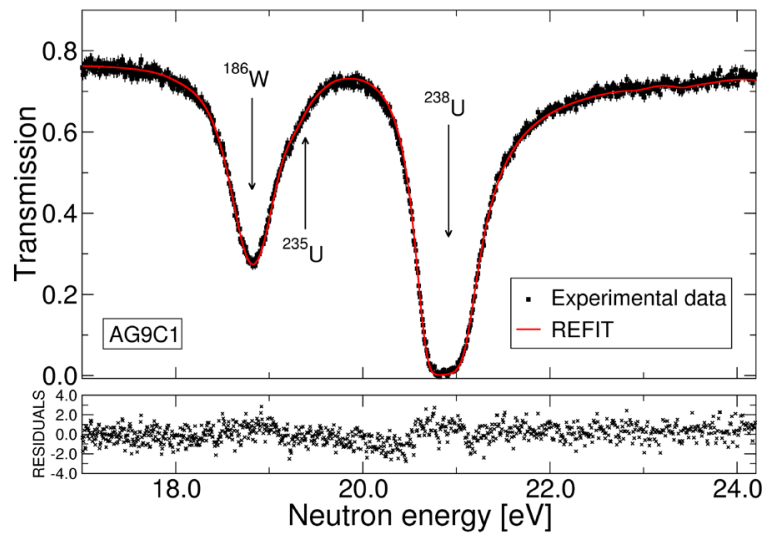

Figure 6. Transmission spectra of the AG9C1 sample in the vicinity of the ${ }^{186} \mathrm{~W}$ resonance at $18.8 \mathrm{eV}$.

\section{Discussion and Conclusions}

As indicated in the Introduction, an interpretation of pile oscillation measurements at the MINERVE facility to determine the reactivity worth of specific elements reveals substantial differences between the calculated and experimental results. The tungsten contamination observed in this work could cause such a difference. This assumption was investigated for the silver samples of the Burn-Up Credit programme (AG9C1, AG9C2 and UNC) by including tungsten in the neutron transport calculations. The reactivity worth in a PWR-UO ${ }_{2}$ lattice was calculated with the PIMS tool [11], using the APOLLO2 deterministic code [12]. The impact of the tungsten content was calculated for the AG9C1 and AG9C2 sample, but also for the reference sample $\mathrm{UNC}$, that only contains pure $\mathrm{UO}_{2}$ pellets without silver. The reactivity worth due to ${ }^{109} \mathrm{Ag}$ is obtained from the measurements with the AG9C1 and AG9C2 samples by subtracting the reactivity worth of the $\mathrm{UO}_{2}$ matrix obtained from the UNC sample. Preliminary results indicate that the tungsten contamination will increase the ratio between the calculated and experimental reactivity worth by $+2.6 \%$ for $\mathrm{AG} 9 \mathrm{C} 1$ and $+5.7 \%$ for AG9C2. These non-negligible corrections make all the results obtained in the framework of the BUC program questionable and a detailed study of the composition of the samples used for the BUC and MAESTRO programmes is recommended. This study could include an analysis by NRTA and even Neutron Resonance Capture Analysis (NRCA), since the latter is more sensitive to the presence of impurities or trace elements.

\section{Acknowledgements}

This work was supported by the EUFRAT open access programme of the Joint Research Centre. Šalamon L. acknowledges the GENTLE project (grant No 323304) for the support during his stay at the JRC Geel.

\section{References}

[1] A. Gruel et al., Nucl. Sci. Eng. 169, 229-244 (2011).

[2] N. Thiollay et al., Int. Conf. Nucl. Criticality Safety (ICNC'99), Versailles, France (1999).

[3] P. Leconte et al., 3rd Intern. Conf. Nucl. Adv. Nucl. Instrum., Meas. Method Appl. (ANIMMA), Marseille, France, DOI: 10.1109/ANIMMA.2013.6728101.

[4] W. Mondelaers and P. Schillebeeckx, Notizario 11, 19-25 (2006).

[5] B. Becker et al., Jour. Instr. 7, P11002 (2012).

[6] P. Schillebeeckx, B. Becker, H. Harada and S. Kopecky, JRC Sci Policy Reports, Report EUR 26848 (2014).

[7] P. Schillebeeckx et al., Nucl. Data Sheets 113, 301543100 (2012).

[8] M. C. Moxon and J. B. Brisland, AEA Techn., AEAInTec-0630 (1991).

[9] X-5 Monte Carlo Team, 'MCNP-A general Monte Carlo N-Particle Transport Code, Version 5', Volume I: Overview and Theory LA-UR-03-1987 (2005).

[10] L. Šalamon et al., Nucl. Instrum. Methods Phys. Res. B 446 19-28 (2019).

[11] H. Derrien, A. Courcelle, L. C. Leal, and A. Santamarina, Nucl. Sci. Eng. 161(2) 131-159 (2009).

[12] D. Bernard and P. Leconte,Proc., Proc. Int. Conf. Nucl. Data for Sci. and Tech., Jeju Island, South Korea (2010), DOI: 10.3938/jkps.59.1119.

[13] R. Sanchez et al., Nucl. Sci. Tech. 42, 474-499 (2010). 